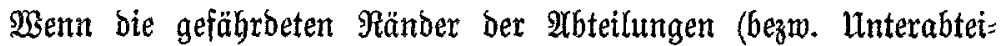
lungen), alfo in ber Regel die Weft= und Sübränder in ăbntidjer Meije behandelt witroen, wie bas eben für ben Malbrand geichildert wurbe, jo

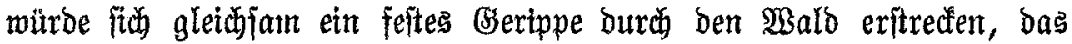
bem gamen Riorper, ber überall burch riditige Pflege gejund und wider:

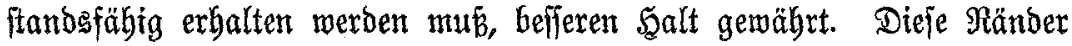

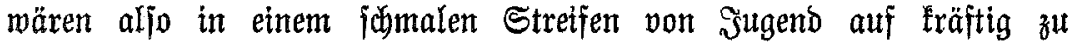
burdjforiten. Wo fajwades Material nidht verwertbar ift, wo zut Durch: forftung foldher $\mathfrak{A} r$ beiten bie Rräfte fehlen - ber Alrbeitermangel broht

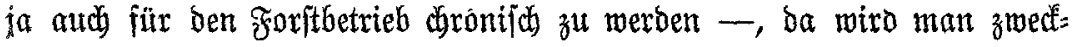

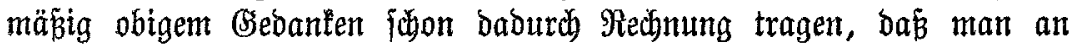

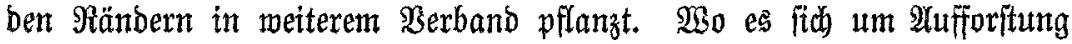
grober אahlflädyen banbelt, auf benen fith 2lbteilung an slbteilung in jofier endlojer Fhlutht reiht, wäre bies wohl ber Errwägung wert.

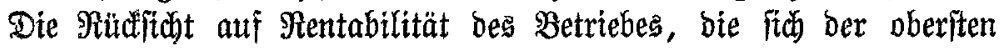
Forberung bex Foritwirtidhaft, Der Radhlaitigfeit, als gleidberedigtigt zur Seite ftellt, verlangt, dapj jeder Beitand im Beitpunft jeiner Siebsreife genukt merbe. Dies ift aber nur bann möglid, wenn ber biebsretife

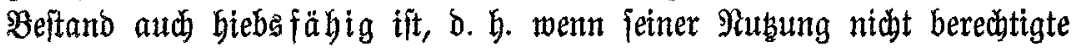
betriebstedgnifche Forberungen entgegenftehen.

Es foll aljo jeder Beftand möglicht felbftändig, möglichft unabjängig

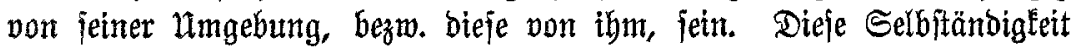
wirb aber erreicht, wenn jeder Beftand einen gefeitigten Fand bejitbt.

Benn idy fo aus ber Fidtenlultur etwas in ba马 Gebiet ber Forft= einrichtung geraten bin, fo wirb man mix bas wogl nicht übel nehmen.

\title{
II. Mitteilumgen.
}

\section{Der deutiche forftnerein und die Colalforftwereine, mit befonderer Bezugnahme auf Bayern.}

Das Berzeidynis ber Mittglieber bes beutichen Foritvereins nach bem Stanbe vom 1. Sanuar 1908, wie basfelbe in ber jüngften Nummer Der Mitteilungen biejes Bereins fich finbet, gibt Belegentheit, über ben Stand bes forftlidjen Bereinswejens in Deutichland fich zu orientieren unb einzelne auffallenbe Errjüeinungen wahrzłnehment.

Utnter ben 1981 Miitgliebern, bie ber beutjâse Froritverein zählt, be= finden fich u. a. 20 sofalforftwereine, 6 פalobefizervereine und 3 Forft= 


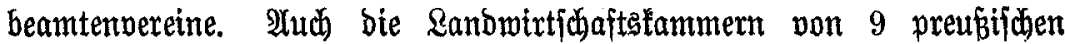
Frovinzen gehören bem $\mathfrak{D}$. F. $\mathfrak{F}$. als Mitglieber an.

Die Sofalvereine fint in jehr unregelmäbigtger Meife über ganz Deutjuland nerteilt; boch befindet fich in jebem ber 16 Ranbesbezinfe

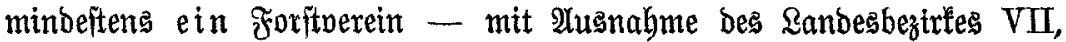
bes jüblichen und füböftlichen Bayern, in B̈egenfas zum \&anbes= bezinf $V$, Şannover und Meftfalen, wofelbft außser 2 Rofalforftwereinen 4 Baldbelïberbereine beftehen und 2 sandwirtichaftstammern aud in Foritjachen tätig îno.

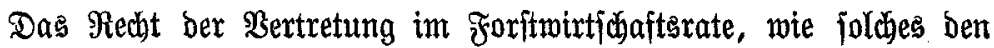

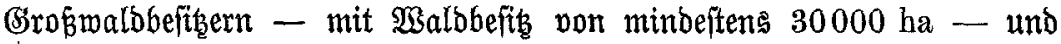

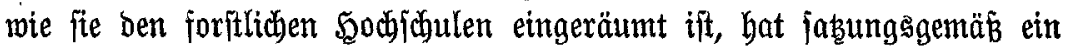
Forftoerein nur bann, wenn er äbnliche 8 medéte verfolgt, wie ber $\mathfrak{D}$. F. $\mathfrak{F}$, wenn er biejem als Mitglieb angebört, und wenn unter jeinen Mit= gliebern eine beftimnte Mindeftzabl, 100 bei einer Mitgliederzahl von mehr als 300, Dem $\mathfrak{D}$. $\mathfrak{F}$. $\mathfrak{N}$. beigetreten ifí.

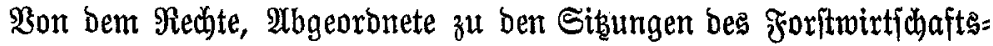
rates abzujenden, wirb von ben Frortwereinen in veridbiedener und wechjelnber Weife (Gebraud gemadht, bäufitg Derart, Dá̉ Dbmänner ober

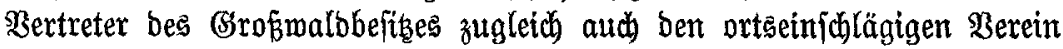
vertreten. \&s find aber unter ben Mitgliebern einer $\mathfrak{I a g u n g}$ bes Farit= mirtichafterates nicht jelten 10 bis 14 Ulfgeoronete pon Foritoereinen anwejent.

Schon aus diejen 2 Angaben geft bervor, baf innerbalb des beutiden Foritpereins und bes Foritmirtichaftsrates biejenigen Ranbesbezirfe ein

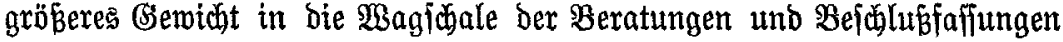
zul legen im ftande fino, welche burch sofalvereine eine veriftärfte $\mathfrak{B e r}=$ tretung befiben.

Mäberer Einblia in Das eingangs erwähnte Mitglieberverzeidntes

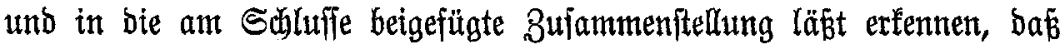
bie 3 ahl ber Mitglieber bes $\mathfrak{D}$. $\mathfrak{F}$. $\mathfrak{B}$. Da am größ̈ten ift, wo auth tätige sotalpereine beiteben.

8weifellos liegt bie Förberung bes foriflichen Bereingewejens in ben einzelnen Bez̧irfen ebenjowohl im S̈ntereffe ber Entwictlung ber Forit= wirtichaft im allgemeinen wie in bem bes $\mathfrak{2}$ aldbefthes und feiner $\mathfrak{B e r}=$ walter.

Sbwohl überall in Deulfolland forftwirtichaftliden und foritwiffen=

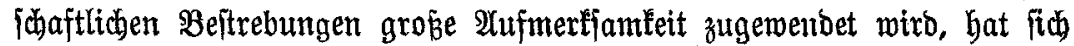
boch bas forftliche $\mathfrak{B}$ ereinswejen in ben einzelnen Rändern und Ranbes= 
teilen verichiebenartig entmiccelelt. Cinen eigentümlichen, nicht nach jeder Rithtung exfreulichen Gang bat bagjelbe in $\mathfrak{B}$ a y ern genommen.

Sa gab eine ßeit, in ber auth bort bas Bebutrfnig und bie Beruffs: frenbe allerorts nach einem 3ufammenfollus brängte unto ihn entweber

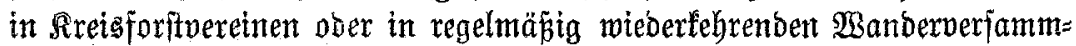
Lungen ber freisangebörigen Forftleute unb $\mathfrak{B a l b b e f t e r}$ fuchte. Bon ben Bereinen mit ftänoiger Mitgliebjoflaft auts jener Beit ijt nur ber \$ffälzer Foritwerein übrig geblieben.

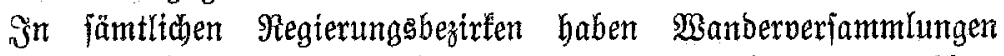
längere Beit geblüht; in Relheim waren 1887 Foritleute aub allen Teilen Bayerns zufammengefommen unb nibht ohne einige \$offnumg auf bie balbige Gribnoung eines bayerijden forftoereines aus= einanbergegangen. Reiber hat fïh biefe Şoffinung niobt erfüfll, und find auch bie Manberwerjammlungen einzelner Sretfe nad) tno nach unter= blieben.

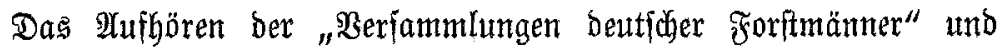
die Entitebung bes "Deutjhen Forftnereing" um bie Sabrhundertwente jeine erite Sauptwerjammlung fand 1900 in 2 Bieshaben ftatt - fonnte ummöğlidh fabulo fein an jenem Erlöfden ber forftlichen Bereinstätigfeit. Satte boch gerade bex beutjide Forftwerein burdh bie bereits ermäbnten

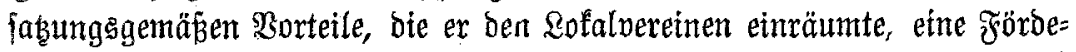
rutng bes foritlithen Bereinswejens in $\mathfrak{A}$ uge gehabt und anderwärts auch bewirft!

(S) mögen berjhiedene $\mathfrak{u}$ riad)en im Spiel gewejen jein, bie bas Snterefie an ber bisherigen $B$ ereinstätigleit in ber Forftwelt bes rechts: rbeinithen Bayern - bei ben Walbbeitien jowohl wie bei ben Forft= beamten - erlahmen ließ̧en. Diefes Şntereffe war bei ben Maldbefizern

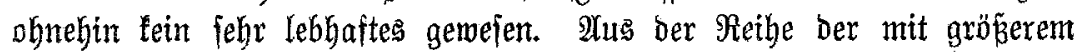

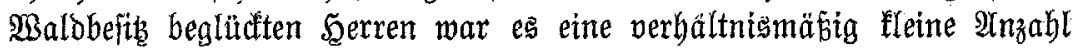

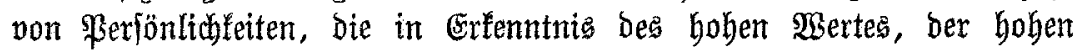
Bebeutung bes $\mathfrak{X a l b e s}$ gerne fich beteiligten, wenn bie sinlabung zut einer foriftliden $\mathfrak{B a n d e r v e r f a m m l a n t}$ an fie herantrat; aber bie $\mathfrak{U} \mathfrak{n}=$ regung mußste von ben Berufsforitleuten auggehen, und bei ber $\mathfrak{B e r}=$ fammlung ftel bie Reitung in ber Regel einem Foritbeamten zu.

Uber aud bie Forftbeamten maren jeit einiger Zeit za Derartigen Beranitaltungen weniger leidht zu baben und mehr geneigt, bie ihnen neben ber bienfitlichen Berufstätigfeit übrige Beit uns Sraft nady einer andern ibnen nabe liegenden ßidatung zu verwenden.

Die $\mathfrak{A}$ usgeftaltung bes foritlichen Unterribtes uno bie Foritorgant= fation vom Sahre 1885 hatte bie längft erhoffite ફ̋lbung bes ganzen 
Faches uno bie gröbere Selbitänbigleit des vermaltenden Frorftbeamten gebracht; auch waren biejelben endlich in Rang unb Gsebaltstlaffe ben Imtšvorftänden anderer Siategorien geidigeitefllt worben.

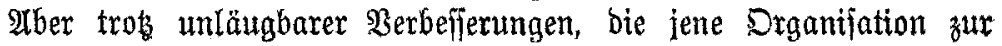
Folge hatte, war bod etne Rethe von Münjon unerfüllt geblieben, nete Slagen traten hervor, bie fich auf bie bientlidie Stellung unb Bejoldung, bie Rage ber Forftamtsaifittenten, bie Entidäbtgung für Dienftauimand

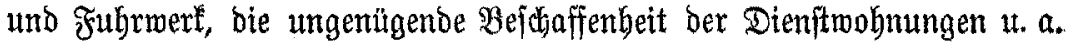
bezogen.

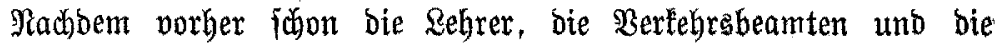
Förfter za Bereinen zur $\mathfrak{B a b}$ arung ibrer Stanbesintereffen fich zufammen= gejolloffen batten, entitano aud ein "Serein bayr. Staatéforfoerwaltungs= beamten", ber ès fich naw ben Sabungen vom 15. Samtar 1904 zur

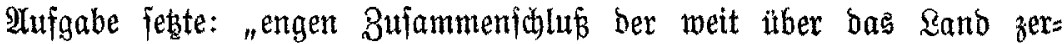

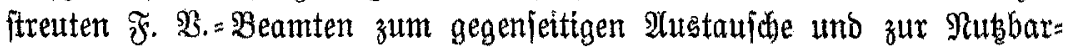

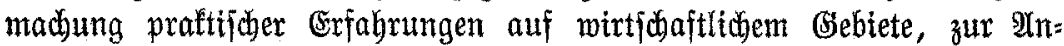

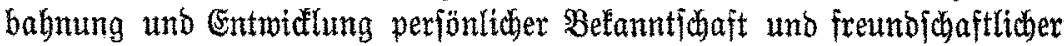
Bezieluungen unter ben Fadgenoffen, fomie zur Mahrung ber Sonder= intereifen."

Diejex Berein, von Dem zwar bie böheren Foritbeamten und bie

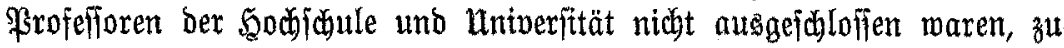
bem fie aber aud nidbt bejonbers herangezogen wurben, hat jebr rajh Berbreitung gefumben, und es gebört ihm zurzeit ber gröfote Teil ber bayr. Forftmeifter, Forftaffefforen, Forftaffiftenten unb :Bermaltunggbienft= Irojpiranten an, im ganzen gegen 900 Mitglteder.

Durd Rreis= uno Bez̧irtsperbände, burch bie regelmäßígen Ber: jammlungen ber leşteren uno bie aljiäbrliche Generalverfammlung it eine bas ganze Rand umfafiende Drganijation geidjaffen, bie zur Er:

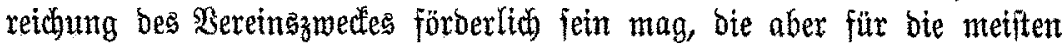
Mittglieber eine anderweitige Betätigung fachmännijłen ßereinglebens

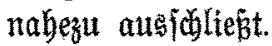

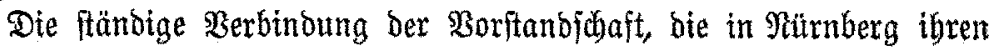
Sith hat, mit ben Mitgliebern und biefer unter fich miro durch bie "Mitteilungen" bes Bereins erhalten, bie in unregelmäß̈̈iger Folge ex=

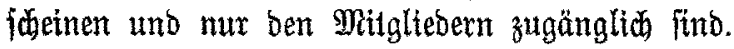

Es ift für ben ferner Stehenoen faber gat erfennen, ob ber Berein

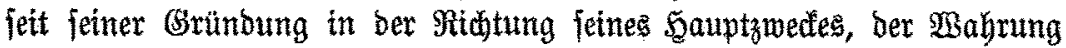
ber Standesintereffen, folon Erflettldes erzitelt hat; bod idheint es fait

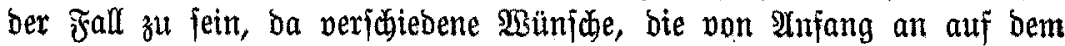
Bereinsprogramm ftanden und in ber vom Bereine ber Staatsregierung 
unterbreiteten Denfichrift ausgejprochen wurben, bereits erfüllt find, und andere - mie zu hoffen - butib bie Dem Randtage zurzeit porliegenden 2Inträge der Staatsregierung iłrer Erfülllung entgegengeben.

Wenn aber über futz ober lang exreidyt fein wirb, was bie baye= rifoden Forftwerwaltungsheamten in Mahrung ihrer Standesintereffen er= ftrebt haben, bann wird und muf boch meeber eine Beit fommen, in ber fte Muke finden und genteigt fein werben, bem forfllichen Bereingmefen im frïheren weiteren Sinne fid zuzuwenden und fid zu freuen ber Mit= arbeit an ber "F̌̉broerung foritlicher Şnterefien, an ber \$flege forftlicher

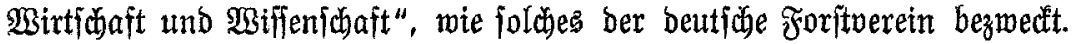

Das erite Beichen ber Bereitwillighteit zu foldher Mitarbeit, ber erite

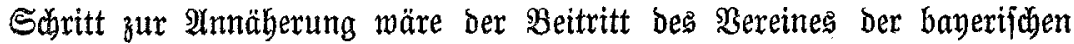
Forftverwaltungsbeamten zum deutfiden Forftuerein als Mitglied bes= jelben.

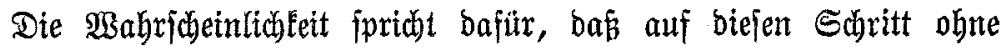

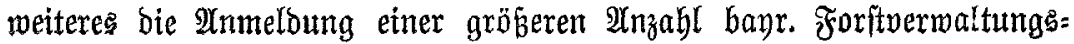
beamten folgen unb bả baburdh ber $\mathfrak{B u n f d h}$ und bie Möglidbleit ge= fördert werben wird, eine Bertretung ihres Bereins im Foritwirtfdaftärate zit extangen.

Bei bem zurzeit unverfennbar auggejprodenen Sharafter bes $\mathfrak{B e r}=$ eines ber bayr. Staatsforitpermaltungsbeamten als Sintereffenten = Berein mürbe biefes Streben auj Sdywierigfeiten ftofen, die wohl mar burd

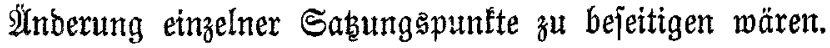

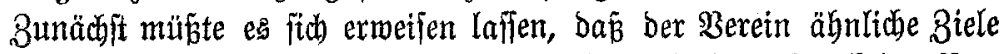

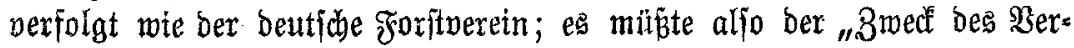
eing" in ben Satzungen bie oben angebeutete Frweiterung eriabren, es

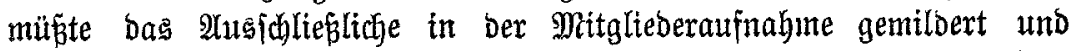
bürfte $\mathfrak{B a l d b e f t h e r n ~ u n d ~} \mathfrak{B a l b f r e u n d e n ~ b e r ~ B e i t r i t t ~ e r m o ̈ g l i c h t ~ w e r b e n . ~}$

Die in ben Sakzungen nicht begründete, bisker aber aus 3 wect:

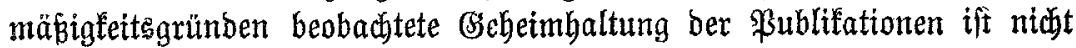
bazu angetan, bem Bereine Zreunbe zu geminnen und feine Birffamfeit nach aupen zu erböben; fie müşte aufbören, fobald ber Serein bie Förobe= nung foritticher sntereffen auf feine Fabne geidgrieben haben wirb.

MSenn bieje Schwiexigfeiten übermunben werben Ëönten und wenn ber Berein, als Berein bayerifber Froritmänner nach Bwed uno $\mathfrak{B i r f e n}$ ben andern Rofalforitwereinen in Deutifland ebenbürtig, feine Stelle im

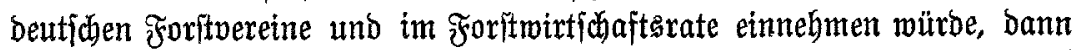
mären bie \$Bünjibe, bie bier mux angeregt weroen wollten, erreidht, wohl aud in Sinne zablretcher Forftleute in Bayern.

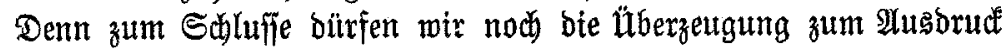




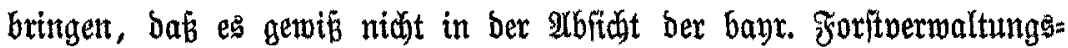
beamten liegt, ftatt bes Mnjwhilfes an bas grop̉e Ganze bemjelben von

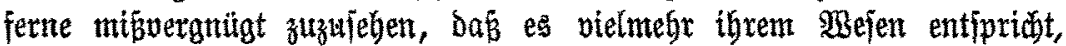
auch in ber Bereingtätigleit fräjtig mitzumirlen an bem Fortforitte umferer beutichen Foritwirtichaft uno Foritmiffenidaft.

Raegfelot.

\section{2us 2Tordamerifa.}

\section{Quterifutifife 5olsontren.}

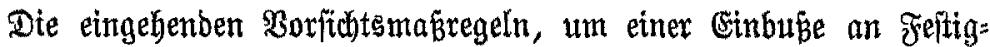

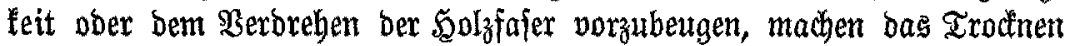

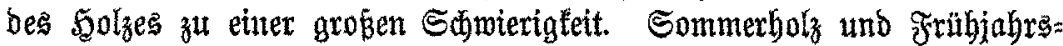

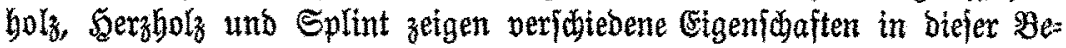
zichung. Şm lebenden $\mathfrak{B a u m}$ wie im grünen Sgolze bilbet $\mathfrak{B a f f e r}$ einen Sauptbeftanotetl, bet ber Troctunng bringt bas Maffer aus ben $3 e l l=$ rãumen burch bie Zellwänoe, bieje felbft bleiben bamit gejăttigt; ift alles

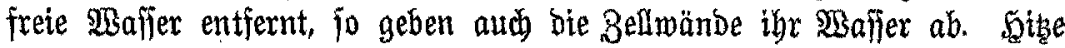
erböht bie 2tbforptionthaft ber Fibern unb unterftübet fo bie $23 a f f e r=$

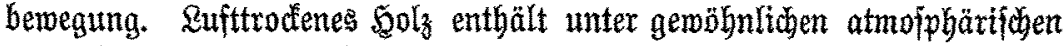
Merbältniffen 10-20\% Fetudtigleit, während gebarrtes ఏolz niaht mehr als $5 \%$ enthält, wenigitens menn es aus ber Darre lommt. Für bie einzelne \$olzart fommen als Bebingungen hierfür in Betracht: Better verbältniffe beim lufttrodenen Buftanbe und Demperatur in ber Darre

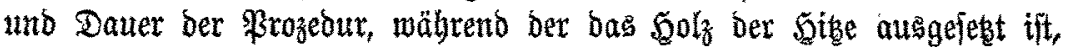
beim Darren Des Golles.

Das fino bie einleitenden Borbemerfungen, bie bent von Frebertat

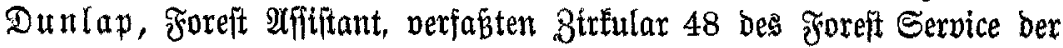
Ber. Staaten Norbamerifas voraniteben, welches fith mit dem Darren bes 50 lazes beidäftigl.

Die geit, bie ber \$rozes ber Dfentrodiung verlangt, ift verfdieden

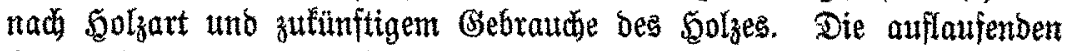
Roften bewegen rich zmifhen weiteften Grenzen, fie betragen felbit für nidjt weit vonteinander fitebende \$srodulte 75 Čents bis 5 Dollars (3 A

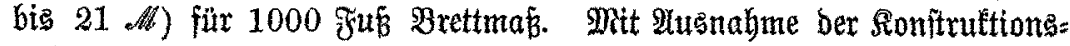
hälzer werben faft alle ફartbölzer (Raubbölzer) gebarrt uno finden fo mannigfaltigite $\mathfrak{B e r w e n b u n g . ~}$

Trodenöfen werben zur gegenmärtigen Beit nach einbeitlthem Primżip

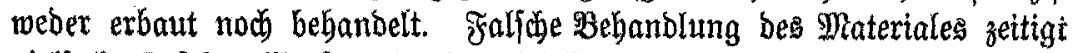

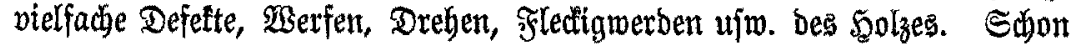

